\title{
Study of Self-organizing Fuzzy Neural Network Control for Flexible Material Path Processing Tangent Following Error Compensation
}

\author{
Yaohua Deng ${ }^{a}$, Xiali Liu ${ }^{b^{*}}$,Liming Wu and Qiaofen Zhang \\ Guangdong University of Technology, Guangzhou, China, 510006 \\ adengyaohua@gdut.edu.cn, ${ }^{\mathrm{b}}$ liuxiali0128@foxmail.com
}

\begin{abstract}
Keywords:Flexible material path processing,Tangent following,Error compensation, Self-organizing fuzzy neural network control

Abstract. The control rules of fuzzy neural network controller for flexible material path processing tangent following are acquired depending on prior knowledge of designers and experts in previous studies, this leads to the difficulty and error of the preliminary calculation. The paper studies and designs self-organizing fuzzy neural network, completes offline training through fuzzy reasoning, realizes the simulation of process knowledge and experience, and the control quantity is given according to the controlled process, consequently the deformation compensation scheme is obtained. The simulation shows that the self-organizing fuzzy neural network controller has better effect on tangent following error compensate for the flexible material path processing .
\end{abstract}

\section{Introduction}

Flexible material path processing refers to the various complex graphics processing on flexible materials and emerging unsmooth stereo pattern on the surface[1,2]. Tangent following refers to constantly adjust the blade position to make the blade and the cutting contour curve remain tangent during flexible material curve cutting operation. Previous studies have shown that flexible material path processing deformation compensation control is mainly predicted compensation quantity based on fuzzy neural network so that automatic deformation compensation control achieved in the process $[3,4]$. The control rules of a fuzzy neural network controller are acquired depending on prior knowledge of designers and experts, the optimal controller performance can not be guaranteed. In view of the problems existed in the model, this paper integrates the advantages of heuristic self-organization induction, fuzzy and self-learning neural network, studies and designs self-organizing fuzzy neural network, sums up the small and widely representative (rule coverage) deformation compensation control rule set, and also can adjust compensation scheme according to the measurement of machining status information feedback.

\section{Tangent following error compensation self-organizing fuzzy neural network structure}

Based on the self-organizing fuzzy controller[5], neural network and fuzzy control are combined, and a self- organizing fuzzy controller based on neural network is proposed. Model diagram is shown in Fig. 1. Flexible material processing deformation is affected by many factors. By analyzing the importance of these factors,we concluded that the importance of deformation affecting factor of primitive Angle error is greatest[4], so machining angle error and angle error rate are chosen as the model input, offset angles are outputted through the processing path deformation compensation control model. In Fig. 1, FRNC is a fuzzy inference controller. Its function is parallel fuzzy quantitative reasoning, fuzzy rules, defuzzification and average decision. Finally, the fuzzy control is given.PNN is performance measurement pointer network, replacing performance measurement, control quantity correction of original self-rganizing fuzzy controller model. Probabilistic neural network (PNN) memory performance measurement decision table(PMDT) by off-line training, improve FRNC network online learning signal,and PNN is trained offline by genetic algorithm, Kalman filtering algorithm[6,7] is used as a control quantity correction online learning algorithm. 


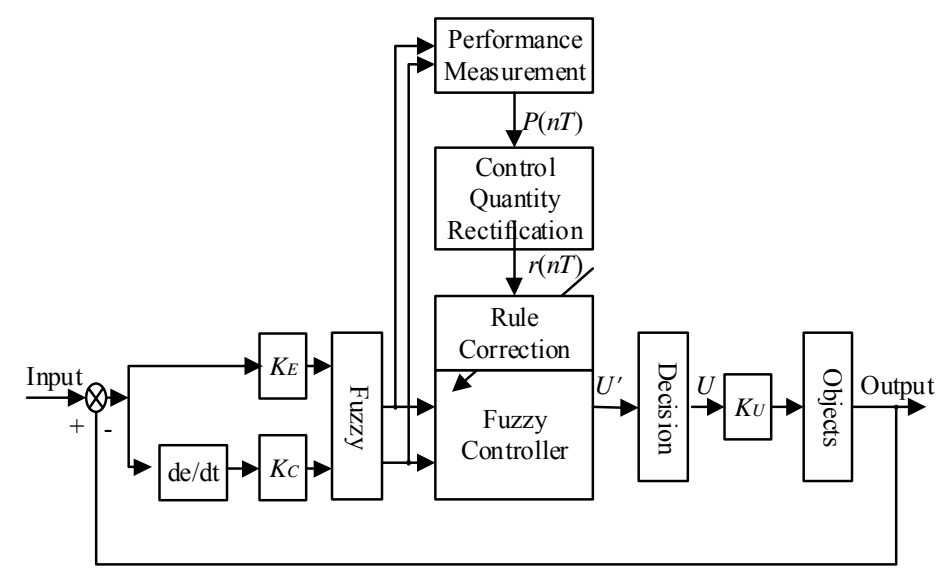

Fig. 1. Self-organizing fuzzy neural network

\section{Design of the tangent following error compensation self-organizing fuzzy neural network controller}

PNN network in self-organizing fuzzy neural networks controller is used to memory the performance measurement index. Genetic algorithm is used as the off-line training algorithm of PNN network, the parameters of BP neural network: $P=2 *(2+6)=16$. The input sample value of the PNN network is $(E, E C) \rightarrow \delta u$,After the completion of the PNN network offline training, self-organization fuzzy controller is accessed as a PMDT, which provides learning signal $\delta u$ for FRN network.

$\operatorname{Define}_{r}, \varphi$ and $\delta$ as certain tangential angle(the acute angle between the tangent of curve and horizontal line), actual processing curve tangential angle and offset angle in the flexible material path processing control; Let $e=\varphi-\varphi_{r}$ be processing angle error, $e c=\mathrm{de} / \mathrm{dt}$ be variation rate of angle error; $k_{e}, k_{e c}$ and $k_{\delta}$ is quantization factor of e, ec and scale factor of $\delta$. Fuzzy control has three main parts: fuzzy, fuzzy rules and anti-fuzzy, fuzzy rules fuzzy and anti-fuzzy are achieved by fuzzy neural network. Performance measurement, control quantity rectification and rule correction conpose a standard self-organizing controller. Conditional inference rules of fuzzy logic control can be expressed as:

$$
\begin{aligned}
& R_{i}: \operatorname{IF}\left(x_{i} \text { is } A_{i 1}\right) \text { and } \mathrm{L} \text { and }\left(x_{n} \text { is } A_{i n}\right) \\
& \operatorname{THEN}\left(\delta \text { is } B_{i}\right)
\end{aligned}
$$

Simplifying the reasoning conclusion:

$$
\delta^{*}=\sum_{i=1}^{n} \alpha_{i} B_{i} / \sum_{i=1}^{n} \alpha_{i}, \alpha_{i}=\prod_{j=0}^{n} \mu_{A_{i j}}\left(x_{j}\right)
$$

$R_{i}$ is ith control rule, $x_{j}=(j=i, \mathrm{~L} n)$ is fuzzy input variable, $A_{i j}$ is a fuzzy subset of membership function $\mu_{A_{i j}}\left(x_{j}\right), \delta$ is fuzzy control variable, $B_{i}$ is control quantity of rule.

Fuzzy neural network controller for fuzzy and anti-fuzzy is shown in Fig. 2.

In flexible material processing direction, $\mathrm{e}, \mathrm{ec}$ and $\delta$ are in the range of $\left[-90^{\circ},+90^{\circ}\right],\left[-90^{\circ},+90^{\circ}\right]$ and $\left[-15^{\circ},+15^{\circ}\right]$ respectively(Clockwise direction is positive), then quantify the range as 13 levels from -6 to $6 . E^{\prime \prime}, E C^{\circ}$ and $\delta^{\circ}$ are fuzzy quantity of e, ec and $\delta$, their fuzzy subsets are $N B, N M, N S, Z O, B P, P M$, and $P B$. Fuzzy membership function of $E^{\prime}, E C^{\prime}$ and $\delta^{0}$ are shown in Fig. 3. After neural network trained by off-line learning, adjust $v_{j i}, v_{k j}$ to complete the fuzzy control rules. 


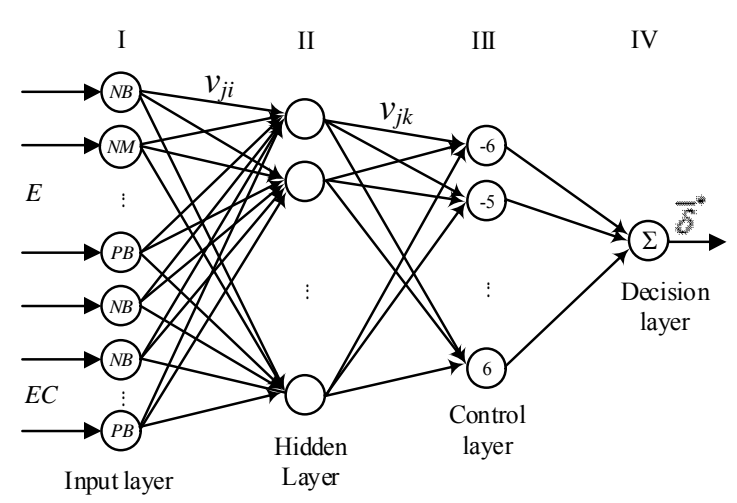

Fig. 2. Fuzzy neural network controller

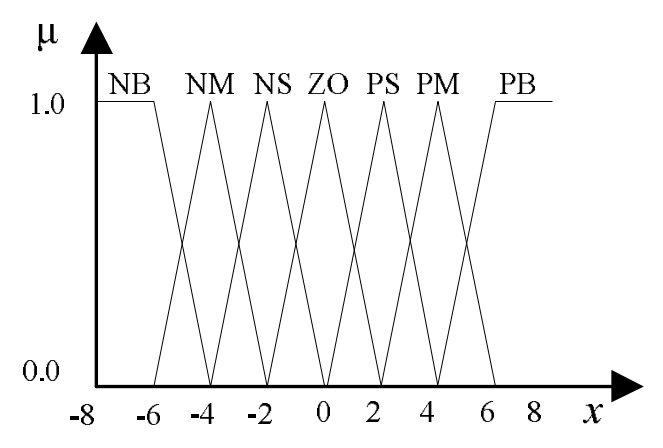

Fig. 3 Fuzzy membership functions

Performance measurement is to measure output characteristics of system, compare the output characteristics and the desired properties, judge whether there is deviation between the two, calculates required rectification quantity to adjust output characteristics[8].

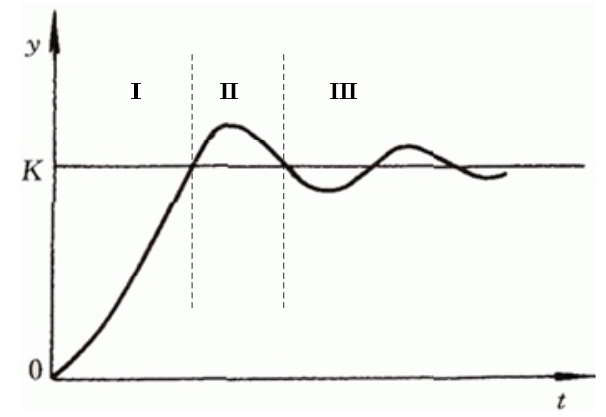

Fig. 4. System output response curve

Fig. 4 shows the system unit step output curve, according to the experience of flexible material processing, Corresponding to different states of $E^{\prime \prime}, E C^{Q}$ on system output response curves, so $P /$ 'should also be taken differently. When the system output response is located at areas I, the actual output of the system is less than the expected output, $E^{\circ}-N M, E C=P S, P=P S$ It indicates that the system output characteristic rises slowly. When the system output response is located at areas II,

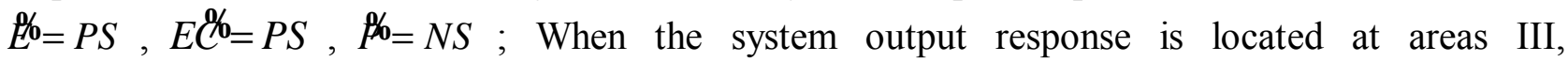

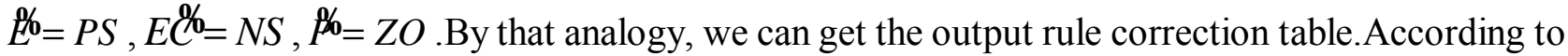
the output rule correction table and the range of $E^{2}, E \mathcal{C}^{\prime}$ and $P^{\beta}$ 'from -6 to +6 , Output characteristic correction table(Table.1) can be geted.

Table. 1. Output characteristic correction table

\begin{tabular}{|c|c|c|c|c|c|c|c|c|c|c|c|c|c|c|}
\hline$\widetilde{P}$ & \multicolumn{10}{|c|}{$\widetilde{E}$} \\
\hline$E \widetilde{C}$ & -6 & -5 & -4 & -3 & -2 & -1 & 0 & 1 & 2 & 3 & 4 & 5 & 6 \\
\hline-6 & 6 & 6 & 6 & 6 & 5 & 5 & 0 & 0 & 0 & 0 & 0 & 0 & 0 \\
\hline-5 & 6 & 6 & 6 & 5 & 4 & 4 & 0 & 0 & 0 & 0 & 0 & 0 & 0 \\
\hline-4 & 6 & 6 & 5 & 4 & 3 & 2 & 0 & 0 & 0 & 0 & 0 & 0 & 0 \\
\hline-3 & 6 & 6 & 5 & 4 & 3 & 2 & 0 & 0 & 0 & -2 & -2 & -2 & 0 \\
\hline-2 & 6 & 6 & 5 & 4 & 1 & 1 & 0 & 0 & 0 & -2 & -4 & -2 & 0 \\
\hline-1 & 5 & 5 & 4 & 3 & 2 & 1 & 0 & 0 & 0 & -3 & -5 & -3 & 0 \\
\hline 0 & 4 & 3 & 3 & 3 & 2 & 1 & 0 & -1 & -2 & -4 & -4 & -6 & -6 \\
\hline 1 & 2 & 3 & 4 & 3 & 0 & 0 & 0 & -1 & -2 & -4 & -6 & -6 & -6 \\
\hline 2 & 0 & 2 & 3 & 2 & 0 & 0 & 0 & -1 & -2 & -4 & -6 & -6 & -6 \\
\hline 3 & 0 & 2 & 2 & 2 & 0 & 0 & 0 & -2 & -3 & -4 & -6 & -6 & -6 \\
\hline 4 & 0 & 0 & 0 & 0 & 0 & 0 & 0 & -3 & -4 & -5 & -6 & -6 & -6 \\
\hline 5 & 0 & 0 & 0 & 0 & 0 & 0 & 0 & -4 & -5 & -5 & -6 & -6 & -6 \\
\hline 6 & 0 & 0 & 0 & 0 & 0 & 0 & 0 & -5 & -6 & -6 & -6 & -6 & -6 \\
\hline
\end{tabular}


Self organizing fuzzy control system has some delay. let the system time delay is $m$, New control quantity change can be achieved at the moment $(n T-m T)$ by $\gamma / p n T)$ rectification. This improves the system performance at the moment, the specific expression is:

$$
\delta \%(n T-m T)=\delta \%(n T-m T)+\gamma(p n T)
$$

Through the online learning of fuzzy neural network controller, the weights $v_{j i}, v_{k j}$ and $v_{o k}$ are adjusted, the desired output is adjusted by the output $\delta / c_{\text {of }}$ the controller adaptively with the change of environment, so the tangent following process control of flexible materials is completed.

\section{Simulation tests and results}

The biggest feature of self-organizing fuzzy neural network controller is it still can provide a good adaptive control. when the controller's external environment changes. Control system response is monitored by PNN network according to $E(n T)$ and $E C(n T)$ of the system, after reasoning, a correction amount $\delta u$ is given which is FRN network learning signal to rectify network weights, makes the FRN network output $u^{*}$ approximation to the expected output $u(n T)$.

The specific steps of self-organizing fuzzy neural network control process are as follows:

(1) Train Offline of PNN network, memory performance measurement pointer;

(2) Preset initial weights of FRN network, train offline FRN network through the Kalman filtering algorithm;

(3) Take sample $\{y d(t), y(t)\}$, calculate $e(t)$ and $e c(t)$;

(4) Calculate the network control quantity $u^{*}(t)$ by Kalman filter equation, $u(t)=K_{p}{ }^{*} u^{*}(t)$;

(5) Calculate the network correction control quantity, rectify .

(6) Take $\delta u(t)$ as the learning signal, correct the weights of FRN network, find out the expected control quantity $u(n T)$;

(7) If $J<\varepsilon$, then stop online learning, go to step(3); otherwise, continue to learn, go to step(6).

In Fig. 5, the black curve is trajectory curve to be processed, the red line is control polygon.Fig. 6 is tangential angle curve without compesation in the process of flexible material, Fig. 7 is Tangential angle curve of self-organizing fuzzy neural network controller. From Fig. 5, Fig. 6 and Fig. 7, the controller responds to the deviation, and the corresponding compensation angle is compensated for the flexible material processing system when there is a deviation between the actual machining angle and the preset angle.

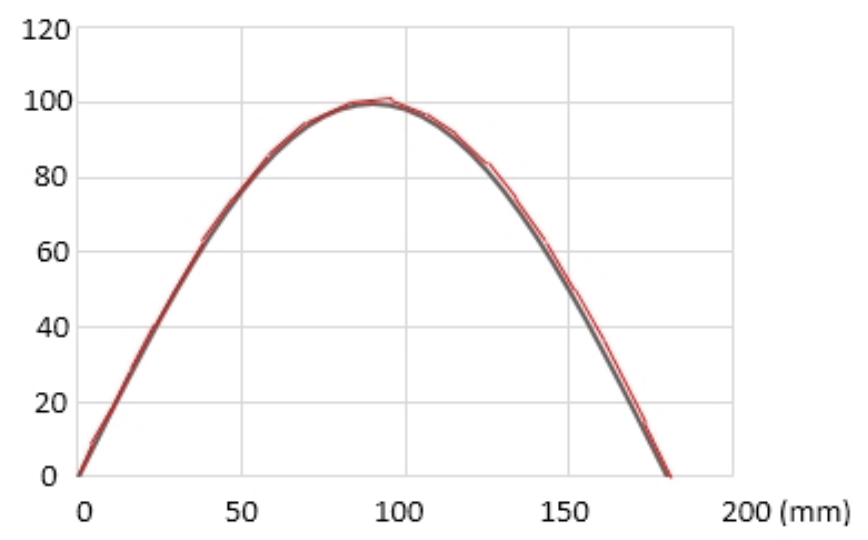

Fig. 5. Trace curve to be processed 


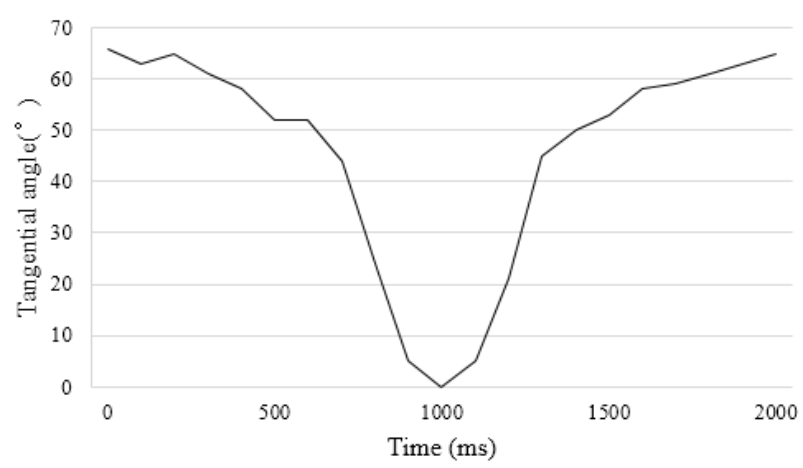

Fig. 6. Tangential angle curve without compesation

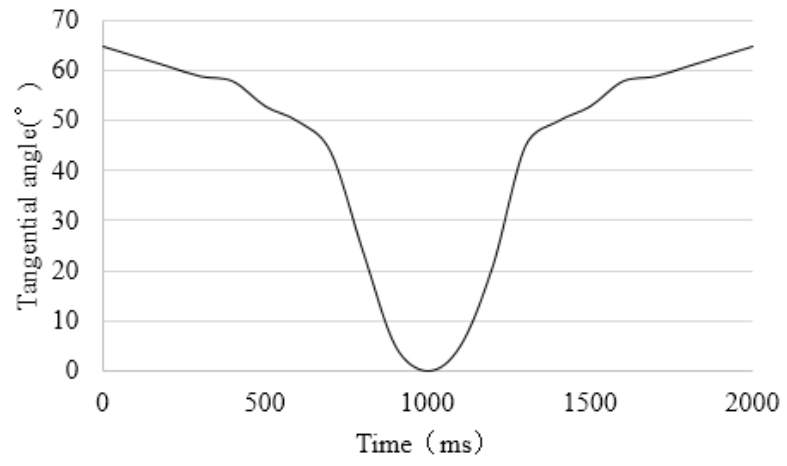

Fig. 7.Tangential angle curve with compesation

\section{Conclusion}

In this paper, a self-organizing fuzzy control strategy based on neural network is proposed, which is applied to the flexible material processing path tangent following error compensation control.

(1) The controller do offline training through fuzzy reasoning,realize the simulation of process knowledge and experience and the control quantity is given according to the controlled process,so the control effect is further improved.

(2) Make the knowledge base of control system compose and update by PNN network, performance measurement indexes will be memoried by genetic algorithm off-line training, then they are accessed to control system for online learning, take the output of PNN network as FRNC learning signal. The validity of the model is verified by simulation.

\section{Acknowledgements}

This work is partially supported by Natural Science Foundation of Guangdong Province, China (no. S2013010013288), Science and Technology Planning Project of Guangdong Province, China(2014A010104012), and National Natural Science Foundation of China (no. 51205069). Thanks for the help.

\section{References}

[1] Yaohua Deng, Sicheng Chen, Bingjing Li, Jiayuan Chen, Liming Wu:submitted to Mathematical Problems in Engineering, (2013).

[2] Yaohua Deng, Jiayuan Chen, Xiali Liu, Sicheng Chen, Qiaofen Zhang, Liming Wu:submitted to Optik - International Journal for Light and Electron Optics. (2014)

[3] Yaohua Deng,Guixiong Liu:submitted to Journal of South China University of Technology ( Natural Science Edition)(2012).

[4] Yaohua Deng,Sicheng Chen, Jiayuan Chen, Jiayi Wu, Qiaofen Zhang, Liming Wu, Hui Chen: Submitted to Journal of Vibroengineering (2014)

[5] Zhao Liang, Guoqiang Zheng,Jishun Li:submitted to Fire Control \& Command Control(2013)(In Chinese)

[6] Yao Wu, Xionglin Luo ,Robustness submitted to Acta Automatica Sinica (2012) (In Chinese)

[7] Jun Kong,Xinyi Tang,Min Jiang et al:submitted to Infrared Millim. Waves(2011) (In Chinese) 